\title{
$H$-supplemented modules with respect to images of fully invariant submodules
}

\author{
A. R. Moniri Hamzekolaee ${ }^{1}$ (1) orcid.org/0000-0002-2852-7870 \\ T. Amouzegar ${ }^{*}$ D orcid.org/0000-0002-0600-5326
}

\author{
${ }^{1}$ University of Mazandaran, Dept. of Mathematics, Babolsar, Iran. \\ a.monirih@umz.ac.ir \\ ${ }^{2}$ Quchan University of Technology, Dept. of Mathematics, Quchan, Iran. \\ Qt.amouzgar@qiet.ac.ir
}

\section{Received: April 2019 | Accepted: October 2020}

\begin{abstract}
:
Lifting modules plays important roles in module theory. H-supplemented modules are a nice generalization of lifting modules which have been studied extensively recently. In this article, we introduce a proper generalization of $\mathrm{H}$-supplemented modules via images of fully invariant submodules. Let $F$ be a fully invariant submodule of a right Rmodule $M$. We say that $M$ is IF - $H$-supplemented in case for every endomorphism $\varphi$ of $M$, there is a direct summand $D$ of $M$ such that $\varphi(F)+X=M$ if and only if $D+X=M$, for every submodule $X$ of $M$. It is proved that $M$ is $I_{F}-H$-supplemented if and only if $F$ is a dual Rickart direct summand of $M$ for a fully invariant noncosingular submodule $F$ of $M$. It is shown that the direct sum of $I_{F}-H$ supplemented modules is not in general $I_{F}-H$-supplemented. Some sufficient conditions such that the direct sum of $I_{F}-H$-supplemented modules is $I_{F}-H$ supplemented are given
\end{abstract}

Keywords: $H$-supplemented module; $\mathrm{I}_{\mathrm{F}}$-lifting module; $\mathrm{I}_{\mathrm{F}}-\mathrm{H}$-supplemented module; Dual Rickart module; Endomorphisms ring.

MSC (2020): 16D10, 16D80.

\section{Cite this article as (IEEE citation style):}

A. R. Moniri Hamzekolaee, and T. Amouzegar, "H-supplemented modules with respect to images of fully invariant submodules", Proyecciones (Antofagasta, On line), vol. 40, no. 1, pp. 35-48, 2021, doi: 10.22199/issn.0717-6279-2021-01-0003

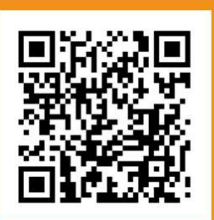

Article copyright: @ 2021 A. R. Moniri Hamzekolaee and T. Amouzegar. This is an open access article distributed under the terms of the Creative Commons License, which permits unrestricted use and distribution provided the original author and source are credited.

*Corresponding author 


\section{Introduction}

All rings considered in this article are associative with an identity element and all modules are unitary right modules unless otherwise stated. Let $R$ be a ring and $M$ a right $R$-module. The ring of all $R$-endomorphisms of $M$ is denoted by $S=\operatorname{End}_{R}(M)$. We use the notation $N \ll M$ to indicate that $N$ is small in $M$ (i.e., for all $L<M, L+N \neq M$ ). A module $M$ is called hollow if every proper submodule of $M$ is small in $M \cdot \operatorname{Rad}(M)$ and $\operatorname{Soc}(M)$ denote the radical and the socle of a module $M$, respectively. A submodule $N$ of $M$ is called a fully invariant submodule of $M$ if for all $\phi \in \operatorname{End}_{R}(M), \phi(N) \subseteq N$.

Let $L \subseteq K \leq M$. We recall that $K$ lies above $L$ in $M$, if $K / L \ll M / L$. A module $M$ is called lifting if every submodule $A$ of $M$ lies above a direct summand $D$ of $M$. A submodule $N$ of $M$ is called supplement in $M$ if there exists a submodule $K$ of $M$ such that $M=N+K$ and $N \cap K \ll N$. A module $M$ is said to be supplemented if every submodule of $M$ has a supplement [4].

Recall that a module $M$ is called $H$-supplemented in case for every submodule $N$ of $M$, there exists a direct summand $D$ of $M$ such that $M=N+X$ if and only if $M=D+X$ for every submodule $X$ of $M$ [9]. In [7], the authors presented some equivalent conditions for a module to be $H$-supplemented that shows that this class of modules is closely related to the concept of small submodules. In [3], the authors introduced a new generalization of $H$-supplemented modules that is Goldie*-supplemented modules via an equivalence relation namely $\beta^{*}$. Let $X$ and $Y$ be submodules of $M$. Then $X \beta^{*} Y$ in $M$ provided $(X+Y) / X \ll M / X$ and $(X+Y) / Y \ll$ $M / Y$. Here it is convenient to state that $M$ is $H$-supplemented if and only if for each submodule $X$ of $M$ there exists a direct summand $D$ of $M$ such that $X \beta^{*} D$ in $M$.

Recall from [8], that a module $M$ is dual Rickart in case, for every endomorphism $\varphi$ of $M$, the image of $\varphi$ is a direct summand of $M$. The author in [1] introduced a generalization of both lifting modules and dual Rickart modules as $\mathcal{I}$-lifting modules. The author showed that a projective $\mathcal{I}$-lifting module is a direct sum of cyclic modules. In [10], the authors studied $H$-supplemented modules via homomorphisms, which generalizes both $H$-supplemented modules and $\mathcal{I}$-lifting modules. They called a module $M$, endomorphism $H$-supplemented ( $E$ - $H$-supplemented, for short) provided for every $\varphi$ in $\operatorname{End}_{R}(M)$, there is a direct summand $D$ of $M$ such that $\operatorname{Im} \varphi \beta^{*} D$. 
The present authors, in [2] introduced a new generalization of $\mathcal{I}$-lifting modules via image of fully invariant submodules. Let $M$ be a module and let $F$ be a fully invariant submodule of $M$. Then $M$ is called $\mathcal{I}_{F}$-lifting in case for every endomorphism $\varphi$ of $M$, there is a direct summand $D$ of $M$ contained in $\varphi(F)$ such that $\varphi(F) / D \ll M / D$. Some properties of $\mathcal{I}_{F}$-lifting modules were investigated in [2].

Inspired by mentioned works on lifting modules and $H$-supplemented modules via a homological approach, we are interested to study on $\mathrm{H}$ supple-mented modules via image of fully invariant submodules. In fact, in the definition of an $E$ - $H$-supplemented module, one can replaced $M$ by a fully invariant submodule of $M$. Let $M$ be a module and let $F$ be a fully invariant submodule of $M$. We say $M$ is $\mathcal{I}_{F}-H$-supplemented provided for every endomorphism $\varphi$ of $M$ there is a direct summand $D$ of $M$ such that $\varphi(F)+X=M$ if and only if $D+X=M$, equivalently $\varphi(F) \beta^{*} D$. In what follows by $F$ we mean a fully invariant submodule of $M$.

One preference of this generalization of the $H$-supplemented modules over other generalizations is that the fully invariant submodules form a complete modular sublattice of the lattice of submodules and they are well mannered with respect to endomorphisms. Many of the important submodules of a module are fully invariant submodules such as the Jacobson radical of a module, the socle of a module, the singular submodule, the cosingular submodule, etc.

In Section 2, we show that $\mathcal{I}_{F-H}-$-supplemented modules are proper generalization of both $\mathcal{I}_{F}$-lifting modules and $H$-supplemented modules. We present some conditions under which the two concepts of $\mathcal{I}_{F}$-lifting and $\mathcal{I}_{F}-H$-supplemented coincide. We also study homomorphic images of $\mathcal{I}_{F}-H$-supplemented modules.

In Section 3, we give an example that shows a finite direct sum of $\mathcal{I}_{F^{-}} H$ supplemented modules is not $\mathcal{I}_{F}-H$-supplemented, in general. We introduce the concept of relative $\mathcal{I}_{F}-H$-supplemented property and use this concept to investigate finite direct sums of $\mathcal{I}_{F-H}-$-supplemented modules.

\section{2. $\mathcal{I}_{F}-H$-supplemented modules}

In this section we introduce $\mathcal{I}_{F}-H$-supplemented modules as a proper generalization of both $\mathcal{I}_{F}$-lifting modules and $H$-supplemented modules. Examples are provided to show that the concept of an $\mathcal{I}_{F}$-H-supplemented module is distinct from both an $\mathcal{I}_{F}$-lifting module and an $H$-supplemented module. As we state in the introduction, we provide conditions under which 
the two concepts of $\mathcal{I}_{F}$-lifting and $\mathcal{I}_{F}$ - $H$-supplemented coincide.

Definition 2.1. Let $M$ be a module and let $F$ be a fully invariant submodule of $M$. We say $M$ is $\mathcal{I}_{F-} H$-supplemented if for every $\varphi \in \operatorname{End}_{R}(M)$, there exists a direct summand $D$ of $M$ such that $\varphi(F)+X=M$ if and only if $D+X=M$ for every submodule $X$ of $M$.

It is clear that every $H$-supplemented module is $\mathcal{I}_{F}$-H-supplemented but the converse is not true (see Example 2.12). Obviously, the sentences " $M$ is $E$ - $H$-supplemented" and " $M$ is $\mathcal{I}_{M-H} H$-supplemented" are the same. Note that every module $M$ is clearly $\mathcal{I}_{0}-H$-supplemented.

It is proved in [7] that a module $M$ is $H$-supplemented if and only if for every submodule $N$ of $M$ there is a direct summand $D$ of $M$ such that $(N+D) / D \ll M / D$ and $(N+D) / N \ll M / N$, i.e., $N \beta^{*} D$. The same is true for $\mathcal{I}_{F-} H$-supplemented modules. One can easily check the following:

Proposition 2.2. The following sentences are equivalent for a module $M$ :

(1) $M$ is $\mathcal{I}_{F}-H$-supplemented;

(2) For every $\varphi \in S$, there exists a direct summand $D$ of $M$ such that $\varphi(F) \beta^{*} D$

(3) For every $\varphi \in S$, there exist a direct summand $D$ and a submodule $N$ of $M$ with $\varphi(F) \subseteq N$ and $D \subseteq N$ such that $\frac{N}{D} \ll \frac{M}{D}$ and $\frac{N}{\varphi(F)} \ll \frac{M}{\varphi(F)}$.

Below, we shall provide some examples of $\mathcal{I}_{F}-H$-supplemented modules.

Examples 2.3. (1) Every $\mathcal{I}_{F}$-lifting module is $\mathcal{I}_{F}$ - $H$-supplemented. In particular every lifting module $M$ is $\mathcal{I}_{F}-H$-supplemented for every fully invariant submodule $F$ of $M$.

(2) Let $p$ be a prime number. Then the $\mathbf{Z}$-module $M=\mathbf{Z}_{p^{2}}$ is not a dual Rickart module. Now, $\operatorname{Rad}(M)=(p) \neq 0$. Since $M$ is a hollow module, $M$ is $\mathcal{I}_{\operatorname{Rad}(M)^{-}}$-lifting and hence $\mathcal{I}_{\operatorname{Rad}(M)^{-}}-H$-supplemented.

A module $M$ is called epi-retractable provided every submodule of $M$ is a homomorphic image of $M$ [5]. By [5, Example 2.4], every finitely generated module over an PID is epi-retractable. Note that for an epi-retractable module the two concepts $H$-supplemented and $E-H$-supplemented coincide.

Now it is easy to verify the following proposition:

Proposition 2.4. Let $F$ be a fully invariant submodule of an epi-retractable module $M$. If $M$ is $E$ - $H$-supplemented, then $M$ is $\mathcal{I}_{F}-H$-supplemented. 
We show that the class of $\mathcal{I}_{F}-H$-supplemented modules contains properly the class of $\mathcal{I}_{F}$-lifting modules.

Examples 2.5. (1) Let $p$ be a prime number. Consider the $\mathbf{Z}$-module $M_{1}=\mathbf{Z}_{p^{3}}$. Then by [7, Example 4.6], the $\mathbf{Z}$-module $M=M_{1} \oplus \frac{M_{1}}{(p)} \oplus \frac{(p)}{\left(p^{2}\right)} \oplus$ $\frac{\left(p^{2}\right)}{(0)}$ is $H$-supplemented. Since $\mathbf{Z}_{p} \oplus \mathbf{Z}_{p^{3}}$ is isomorphic to a direct summand of $M, M$ is not lifting from [6, Corollary 2]. Being $M$ a finitely generated $\mathbf{Z}$-module implies that $M$ is epi-retractable by [5, Example 2.4]. Hence $M$ is not $\mathcal{I}$-lifting which means that $M$ is not $\mathcal{I}_{M}$-lifting. In other words, $M$ is $\mathcal{I}_{M}$ - $H$-supplemented as well as $H$-supplemented.

(2) (see [13, Example 2.3]) Let $I$ and $J$ be two ideals of a commutative local ring $R$ with maximal ideal $m$ such that $I \subset J \subseteq m$ and $m J I$ (e.g., $R$ is an $D V R$ with maximal ideal $m, I=m^{4}$ and $J=m^{2}$ ). We consider the module $M=R / I \times R / J$. From [13, Proposition 2.1] it follows that $M$ is $H$-supplemented and so $M$ is $\mathcal{I}_{M}-H$-supplemented. In other words, from [13, Example 2.3], $M$ is not lifting. Being $M$ an epi-retractable module implies $M$ is not $\mathcal{I}$-lifting $\left(\mathcal{I}_{M}\right.$-lifting).

Recall from [12] that an $R$-module $M$ is noncosingular (cosingular) provided $\bar{Z}(M)=M(\bar{Z}(M)=0)$ where $\bar{Z}(M)=\cap\{\operatorname{Kerf} \mid f: M \rightarrow U\}$ for all small $R$-modules $U$.

We present some conditions under which, the two concepts $\mathcal{I}_{F}$-lifting and $\mathcal{I}_{F}-H$-supplemented coincide.

Theorem 2.6. Let $F$ be a fully invariant submodule of a module $M$. If either $F$ is noncosingular or $\operatorname{Rad}(M)=0$, then the following statements are equivalent:

(1) For every $\varphi \in \operatorname{End}_{R}(M)$, the submodule $\varphi(F)$ is a direct summand of $M$;

(2) $M$ is $\mathcal{I}_{F}$-lifting;

(3) $M$ is $\mathcal{I}_{F}-H$-supplemented;

(4) $F$ is a dual Rickart direct summand of $M$.

Proof. We prove when $F$ is noncosingular, the case $\operatorname{Rad}(M)=0$ is the same.

$(1) \Rightarrow(2)$ It is obvious.

$(2) \Rightarrow(3)$ It can be easily verified.

(3) $\Rightarrow$ (4) Let $M$ be $\mathcal{I}_{F}$-H-supplemented and let $\varphi$ be an endomorphism of $M$. Then there is a direct summand $D$ of $M$ such that $\varphi(F) \beta^{*} D$ which means that $(\varphi(F)+D) / D \ll M / D$ and $(\varphi(F)+D) / \varphi(F) \ll M / \varphi(F)$. 
Note that $\varphi(F)$ is noncosingular as well as $F$. It follows that $(\varphi(F)+$ $D) / D \cong \varphi(F) /(\varphi(F) \cap D)$ is a noncosingular submodule of $M / D$. Hence, $\varphi(F)+D=D$ implies that $\varphi(F) \subseteq D$. Now, $D / \varphi(F) \ll M / \varphi(F)$. Set $D \oplus D^{\prime}=M$. Then $D / \varphi(F)+\left(D^{\prime}+\varphi(F)\right) / \varphi(F)=M / \varphi(F)$. Therefore, $D^{\prime}+\varphi(F)=M$. Being $\varphi(F)$ a submodule of $D$ combining with modularity implies $\varphi(F)=D$. Now, let $\psi$ be an endomorphism of $F$. Then $h=j o \psi o \pi_{F}$ is an endomorphism of $M$ where $j$ is the inclusion map and $\pi_{F}$ is the canonical projection. Then $\psi(F)=h(F)$ is a direct summand of $M$. Hence $\psi(F)$ is a direct summand of $F$ showing that $F$ is dual Rickart.

$(4) \Rightarrow(1)$ Let $\varphi$ be an arbitrary endomorphism of $M$. Then $g=\pi_{F} \circ \varphi_{\circ} j$ is an endomorphism of $F$. As $F$ is a dual Rickart module, $g(F)$ is a direct summand of $F$ and also a direct summand of $M$. It follows that $\varphi(F)=$ $g(F)$ is a direct summand of $M$.

Example 2.7. [2, Example 2.8] (1) Let $K$ be a field and $R=\prod_{i=1}^{\infty} K_{i}$ where $K_{i}=K$ for each $i \in \mathbf{N}$.

Let $L$ be an $V$-ring and let $K$ be a field. Then $S=K \times L$ is an $V$-ring as well. Consider the central idempotent $e=(1,0)$ of $S$. Then $S e=e S \cong K$ as both left and right $S$-module. Let $R$ be the ring $M_{n}(S)$ (the ring of all $n \times n$ matrices with entries from $S$ ). As $R$ is Morita-equivalent to $S$, it should be also an $V$-ring. Now, $R$ has a central idempotent, $f=e I$ where $I$ is the identity matrix of $R$. Then $f R=R f$ is isomorphic to $M_{n}(S e)$ so that $f R=R f \cong M_{n}(K)$. Note that $F=R f$ is a two-sided ideal of $R$ and also is a direct summand of $R$. Being $K$ a field implies that $M_{n}(K)$ and hence $F$ is semisimple (dual Rickart). It follows from Theorem 2.6 that $R$ is an $\mathcal{I}_{F^{-}} H$-supplemented module.

Recall from [11] that a module $M$ is weak duo in case every direct summand of $M$ is a fully invariant submodule of $M$. We recall that $L$ is a cosmall submodule of $K$ in $M$ (denoted by $L \stackrel{c s}{\hookrightarrow} K$ in $M$ ) if $K$ lies above $L$ in $M$. Recall that a submodule $L$ of $M$ is called coclosed in $M$, if $L$ has no proper cosmall submodule. It is clear that every direct summand of $M$ is a coclosed submodule of $M$. A module $M$ is said to have cosmall intersection property or $C S I P$ if for any $A, B, C, D \leq M, A \stackrel{c s}{\hookrightarrow} B$ in $M$ and $C \stackrel{c s}{\hookrightarrow} D$ in $M$ imply that $A \cap C \stackrel{c s}{\longrightarrow} B \cap D$ in $M$.

Proposition 2.8. Let $F$ be a fully invariant submodule of a module $M$. If either

(1) $M$ is noncosingular or 
(2) $M$ is a weak duo module or

(3) $M$ is projective or

(4) $M$ has CSIP,

then the two concepts $\mathcal{I}_{F}$-H-supplemented and $\mathcal{I}_{F}$-lifting coincide.

Proof. (1) Let $M$ be $\mathcal{I}_{F-}-H$-supplemented and let $\varphi \in \operatorname{End}_{R}(M)$ be arbitrary. Then by assumption, there is a direct summand $D$ of $M$ such that $(\varphi(F)+D) / D \ll M / D$ and $(\varphi(F)+D) / \varphi(F) \ll M / \varphi(F)$. Since $M$ is noncosingular, $D$ is noncosingular. It follows that $(\varphi(F)+D) / \varphi(F)=0$ which implies that $\varphi(F)+D=\varphi(F)$. So, $D$ is contained in $\varphi(F)$. Therefore, $\varphi(F) / D \ll M / D$. The converse is straightforward.

(2) Let $M$ be an $\mathcal{I}_{F}-H$-supplemented weak duo module and $\varphi \in \operatorname{End}_{R}(M)$. Then there exists a direct summand $D$ of $M$ such that $\varphi(F)+X=M$ if and only if $D+X=M$ for every $X \leq M$. Set $D \oplus D^{\prime}=M$. Then $\varphi(F)+D^{\prime}=M$. As $F$ is fully invariant we have $F=(F \cap D) \oplus\left(F \cap D^{\prime}\right)$. It follows that $M=\varphi\left((F \cap D)+\left(F \cap D^{\prime}\right)\right)+D^{\prime}=\varphi(F \cap D) \oplus D^{\prime}$ (note that $D^{\prime}$ is also fully invariant so that $\left.\varphi\left(F \cap D^{\prime}\right) \subseteq D^{\prime}\right)$. Hence $\varphi(F \cap D)=D$ implies that $D$ is contained in $\varphi(F)$. Suppose that $\varphi(F) / D+L / D=M / D$ for a submodule $L$ of $M$ containing $D$. Then $\varphi(F)+L=M$. Hence $D+L=M$ which implies $L=M$ as required. Therefore, $\varphi(F) / D \ll M / D$.

(3) Similar to the proof of [10, Theorem 2.16].

(4) Let $\phi \in \operatorname{End}_{R}(M)$. Then there exists a direct summand $D$ of $M$ such that $\phi(F) \stackrel{c s}{\hookrightarrow}(\phi(F)+D)$ in $M$ and $D \stackrel{c s}{\hookrightarrow}(\phi(F)+D)$ in $M$. By $C S I P$, $\phi(F) \cap D \stackrel{c s}{\hookrightarrow}(\phi(F)+D)$ in $M$. But $\phi(F) \cap D \subseteq D \subseteq \phi(F)+D$, so $\phi(F) \cap D \stackrel{c s}{\hookrightarrow} D$ in $M$. As $D$ is coclosed in $M, \phi(F) \cap D=D$, hence $D \leq \phi(F)$ and $D \stackrel{c s}{\hookrightarrow} \phi(F)$ in $M$. Therefore $M$ is $\mathcal{I}_{F-\text { lifting. }}$

A characterization of indecomposable $\mathcal{I}_{F}-H$-supplemented modules is presented in the following.

Proposition 2.9. Let $M \neq 0$ be an indecomposable module and let $F<$ $M$ be fully invariant. Then $M$ is $\mathcal{I}_{F}-H$-supplemented if and only if $F \ll M$. In case $F=M$, then $M$ is $\mathcal{I}_{M}-H$-supplemented if and only if every nonzero endomorphism $\varphi$ of $M$ is epimorphism or $\operatorname{Im} \varphi \ll M$.

Proof. Let $M$ be $\mathcal{I}_{F-}-H$-supplemented and let $\imath \in \operatorname{End}_{R}(M)$ be the identity endomorphism. Then there exists a direct summand $D$ of $M$ such that $F+X=\imath(F)+X=M$ if and only if $D+X=M$ for every submodule $X$ of $M$. By assumption, either $D=0$ or $D=M$. Second case will not happen as $F<M$. On the other hand, $D=0$ implies that $F \ll M$. 
The converse is obvious as for every $\varphi \in \operatorname{End}_{R}(M)$, the condition $F \ll M$ implies $\varphi(F)+X=M$ if and only if $0+X=M$, for every $X \leq M$. The latter follows from [10, Proposition 2.3].

Following presents a characterization of an $\mathcal{I}_{F-}-H$-supplemented module $M$ when $F$ is a direct summand of $M$.

Theorem 2.10. Let $F$ be a fully invariant direct summand of a module $M$. If $F$ is $E$ - $H$-supplemented, then $M$ is $\mathcal{I}_{F}-H$-supplemented. The converse holds, in case $M$ is a weak duo module.

Proof. $(\Rightarrow)$ Let $F$ be $E$ - $H$-supplemented and let $\varphi$ be an endomorphism of $M$. Consider $q=\pi_{F} o \varphi o j: F \rightarrow F$, which is an endomorphism of $F$, where $j: F \rightarrow M$ is the inclusion and $\pi_{F}: M \rightarrow F$ is the projection map on $F$. Being $F$ a fully invariant submodule of $M$ implies that $q(F)=\varphi(F)$. As $F$ is $E$ - $H$-supplemented, there is a direct summand $D$ of $F$ (so that of $M)$ such that $q(F)+Y=F$ if and only if $D+Y=F$ for every submodule $Y$ of $F$. Now, suppose that $\varphi(F)+X=M$ for a submodule $X$ of $M$. Then $\varphi(F)+X \cap F=F$. Then, $D+(X \cap F)=F$. It follows that $D+X=F+X=M$. For the converse, let $D+X=M$ where $X \leq M$. Then modularity implies $D+(X \cap F)=F$. Since $F$ is $E$ - $H$-supplemented, $\varphi(F)+(X \cap F)=F$. Hence $\varphi(F)+X=F+X=M$ as required. Therefore, $M$ is $\mathcal{I}_{F}-H$-supplemented.

$(\Leftarrow)$ Let $g: F \rightarrow F$ be an endomorphism of $F$ and $F \oplus F^{\prime}=M$ for a submodule $F^{\prime}$ of $M$. Then $h=j o g o \pi_{F}: M \rightarrow M$ is an endomorphism of $M$ where $j: F \rightarrow M$ is the inclusion and $\pi_{F}: M \rightarrow F$ is the projection on $F$. It is straightforward to check $h(F)=g(F)$. As $M$ is $\mathcal{I}_{F}-H$-supplemented, there exists a direct summand $D$ of $M$ such that $g(F)+X=M$ if and only if $D+X=M$ for every submodule $X$ of $M$. We shall verify $g(F)+Y=F$ if and only if $(F \cap D)+Y=F$. Now, let $g(F)+Y=F$ for $Y \leq F$. Then $g(F)+Y+F^{\prime}=M$. By assumption we have $D+Y+F^{\prime}=M$. By modular law, we conclude that $Y+\left(D+F^{\prime}\right) \cap F=F$. Since $M$ is weak duo, $D$ is a fully invariant submodule of $M$ so that $D=(D \cap F) \oplus\left(D \cap F^{\prime}\right)$. Therefore, $Y+\left[(D \cap F)+F^{\prime}\right] \cap F=F$. Hence $Y+(D \cap F)=F$. The other implication can be verified similarly.

Corollary 2.11. (1) Let $M$ be a module such that $\bar{Z}(M)$ is a direct summand of $M$. If $\bar{Z}(M)$ is $E$-H-supplemented, then $\varphi(\bar{Z}(M))$ is a direct summand of $M$ for every $\varphi \in \operatorname{End}_{R}(M)$. 
(2) Let $M$ be a module such that $\operatorname{Soc}(M)$ is a direct summand of $M$. Then $M$ is $\mathcal{I}_{\text {Soc }(M)^{-H}} H$-supplemented.

Proof. (1) Let $\bar{Z}(M)$ be an $E$ - $H$-supplemented direct summand of $M$. Then by Theorem $2.10, M$ is $\mathcal{I}_{\bar{Z}(M)}-H$-supplemented. Note that since $\bar{Z}(M)$ is a direct summand of $M$, it is noncosinsgular. Therefore, $\varphi(\bar{Z}(M))$ is a direct summand of $M$ for every $\varphi \in \operatorname{End}_{R}(M)$ by Theorem 2.6. (2) It is clear as $\operatorname{Soc}(M)$ is semisimple.

The following example introduces an $\mathcal{I}_{F^{-}} H$-supplemented module which is not $H$-supplemented.

Example 2.12. Let $K$ be a field and $R=K \times K[[x]]$. Then $J(R)=$ $0 \times(x)$. It follows that $R / J(R) \cong K \times\left(\frac{K[[x]]}{(x)}\right)$ is semisimple. Hence $R$ is a commutative semilocal ring with $\operatorname{Soc}(R)=K \times 0$. Let $M=R_{R}^{(\mathbf{N})}$. Then $\operatorname{Rad}\left(R_{R}^{(\mathbf{N})}\right)$ is not small in $R_{R}^{(\mathbf{N})}$ by $[14,43.9]$. Hence, by [14, 42.5], $M=$ $R_{R}^{(\mathbf{N})}$ is not supplemented. So $M$ is not $H$-supplemented. By [2, Proposition

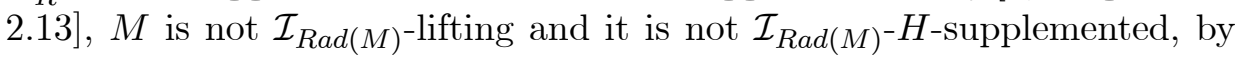
Proposition 2.8(3).

Since $\operatorname{Soc}(M)=K^{(\mathbf{N})}$ is a direct summand of $M$, by Corollary 2.11, $M$ is $\mathcal{I}_{K(\mathbf{N})}-H$-supplemented. Therefore, by Proposition 2.8, $M$ is also $\mathcal{I}_{K(\mathbf{N})}$-lifting.

Proposition 2.13. Let $F$ be a fully invariant submodule of a module $M$ and let $K$ be a fully invariant direct summand of $M$ contained in $F$. If $M$ is $\mathcal{I}_{F-H}-$-supplemented, then $M / K$ is $\mathcal{I}_{F / K}-H$-supplemented.

Proof. Let $g: M / K \rightarrow M / K$ be an endomorphism of $M / K$ and $M=$ $K \oplus K^{\prime}$. Then $f=$ johogo $\pi: M \rightarrow M$ is an endomorphism of $M$. Note that $\pi: M \rightarrow M / K$ is the canonical projection, $h: M / K \rightarrow K^{\prime}$ is the isomorphism induced by the decomposition $M=K \oplus K^{\prime}$ and $j: K^{\prime} \rightarrow M$ is the inclusion. By assuming $g(F / K)=T / K$, one can $f(F)=T \cap K^{\prime}$. Since $M$ is $\mathcal{I}_{F^{-}} H$-supplemented, there is a direct summand $D$ of $M$ such that $\left(T \cap K^{\prime}\right)+X=M$ if and only if $D+X=M$ for every $X \leq M$. Set $M=D \oplus D^{\prime}$. Then $M / K=(D+K) / K+\left(D^{\prime}+K\right) / K$. Since $K$ is a fully invariant submodule of $M$, we have $K=(K \cap D) \oplus\left(K \cap D^{\prime}\right)$. Hence $(D+K) \cap\left(D^{\prime}+K\right)=K$ which implies that $(D+K) / K$ is a direct summand of $M / K$. We shall show that $T / K+Y / K=M / K$ if and only if $(D+K) / K+Y / K=M / K$ for every submodule $Y$ of $M$ containing $K$. In 
first step, let $T / K+Y / K=M / K$. Then $T+Y=M$. As $T$ contains $K$, we have $\left[K+\left(T \cap K^{\prime}\right)\right]+Y=M=\left(T \cap K^{\prime}\right)+Y=M$. Then by assumption, $D+Y=M$. It follows that $(D+K) / K+Y / K=M / K$. In other words, suppose that $(D+K) / K+Y / K=M / K$. Then $D+Y=M$ which implies that $T+Y=M$. Therefore, $T / K+Y / K=M / K$ as required.

\section{Direct sums of $\mathcal{I}_{F}-H$-supplemented modules}

Let $F=\oplus_{i \in I} F_{i}$ where $F_{i}(i \in I)$ is a fully invariant submodule of $M$. The following example shows that a finite direct sum of $\mathcal{I}_{F_{i}}-H$-supplemented modules need not be $\mathcal{I}_{F^{-}} H$-supplemented, in general.

Example 3.1. Let $R$ be a discrete valuation ring and let $I_{1}, \ldots, I_{n}$ be some ideals of $R$. Consider the $R$-module $M \cong R / I_{1} \times \cdots \times R / I_{n}$. Since $R$ is commutative, each $R / I_{i}$ is $H$-supplemented and so each $R / I_{i}$ is $\mathcal{I}_{R / I_{i}}-H$ supplemented. If $I_{1} \subseteq \cdots \subseteq I_{n} \subset R$, then $M$ is $H$-supplemented by [13, Proposition 2.1]. Therefore, $M$ is $\mathcal{I}_{M}-H$-supplemented. Otherwise, i.e., the condition $I_{1} \subseteq \cdots \subseteq I_{n} \subset R$ does not hold, $M$ is not $H$-supplemented. Note also that $M$ is an epi-retractable $R$-module by [5, Example 2.4(3)]. It means that in this case $M$ is not $\mathcal{I}_{M-H} H$-supplemented.

Now we define relative $\mathcal{I}_{F-}-H$-supplemented modules and we apply this concept to study finite direct sums of $\mathcal{I}_{F}-H$-supplemented modules.

Definition 3.2. Let $M$ and $N$ be $R$-modules and let $F$ be a fully invariant submodule of $M$. We say $M$ is $N-\mathcal{I}_{F}-H$-supplemented if for every homomorphism $\phi: M \rightarrow N$, there exists a direct summand $D$ of $N$ such that $\phi(F)+X=N$ if and only if $D+X=N$ for every submodule $X$ of $N$.

It is clear that a module $M$ is $\mathcal{I}_{F^{-}} H$-supplemented if and only if $M$ is $M-\mathcal{I}_{F^{-}} H$-supplemented.

Theorem 3.3. Let $M$ and $N$ be right $R$-modules and let $F$ be a fully invariant submodule of $M$. Then $M$ is $N-\mathcal{I}_{F}-H$-supplemented if and only if for every direct summand $M^{\prime}$ of $M$ and every fully invariant direct summand $N^{\prime}$ of $N, M^{\prime}$ is $N^{\prime}-\mathcal{I}_{F \cap M^{\prime}}-H$-supplemented.

Proof. Let $M^{\prime}=e M$ for some $e^{2}=e \in \operatorname{End}_{R}(M)$, and let $N^{\prime}$ be a fully invariant direct summand of $N$. Then $N=N^{\prime} \oplus T$ for some $T \leq N$. Suppose that $\psi \in \operatorname{Hom}\left(M^{\prime}, N^{\prime}\right)$. We want to show that for any submodule 
$X$ of $N^{\prime}$, there exists a direct summand $D$ of $N^{\prime}$ such that $\psi\left(F \cap M^{\prime}\right)+X=$ $N^{\prime}$ if and only if $D+X=N^{\prime}$.

First note that $e\left(F \cap M^{\prime}\right)=e F$ and $\psi e(F)=\psi\left(F \cap M^{\prime}\right)$. Let $\psi(F \cap$ $\left.M^{\prime}\right)+X=N^{\prime}$ for a submodule $X$ of $N^{\prime}$. Then $\psi e(F)+X+T=N$. Since $\psi e M \subseteq N^{\prime} \subseteq N$ and $M$ is $N$ - $\mathcal{I}_{F}-H$-supplemented, we conclude that $A+X+T=N$ for some direct summands $A$ of $N$. So $X+\left(A \cap N^{\prime}\right)=N^{\prime}$. Note that $A \cap N^{\prime}$ is a direct summand of $N^{\prime}$ as $N^{\prime}$ is fully invariant.

Conversely, assume that $N^{\prime}=A \cap N^{\prime}+X$ where $A \cap N^{\prime}$ is a direct summand of $N^{\prime}$ and $X \leq N^{\prime}$. Then $N=N^{\prime}+T=A \cap N^{\prime}+X+T$. Since $A \cap N^{\prime}$ is a direct summand of $N$ and $M$ is $N-\mathcal{I}_{F}-H$-supplemented, $N=\psi e(F)+X+T$. Hence $N^{\prime}=\psi e(F)+X$, and so $N^{\prime}=\psi\left(F \cap M^{\prime}\right)+X$. Therefore $M^{\prime}$ is $N^{\prime}-\mathcal{I}_{F \cap M^{\prime}}-H$-supplemented. The other side of this theorem is clear.

Corollary 3.4. Let $M$ be $R$-module and let $F$ be a fully invariant submodule of $M$. Then the following condition are equivalent: (1) $M$ is $\mathcal{I}_{F-H}-$ supplemented; (2) For any fully invariant direct summand $N$ of $M$, every direct summand $L$ of $M$ is $N-\mathcal{I}_{F \cap L}-H$-supplemented.

Corollary 3.5. Let $F$ be a fully invariant submodule of a module $M$ and

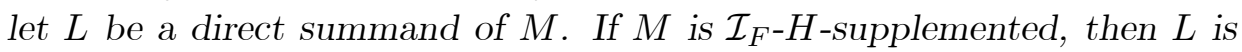
$\mathcal{I}_{F \cap L}-H$-supplemented.

Recall that a module $M$ is said to have the summand sum property (SSP) if the sum of any two direct summands is a direct summand of $M$.

Theorem 3.6. Let $M=\oplus_{i=1}^{n} M_{i}$ and $N$ be right $R$-modules and let $F$ be a fully invariant submodule of $M$. If $N$ has the $S S P$, then $M=\oplus_{i=1}^{n} M_{i}$ is $N$ - $\mathcal{I}_{F}-H$-supplemented if and only if $M_{i}$ is $N$ - $\mathcal{I}_{F \cap M_{i}}-H$-supplemented for all $i \in\{1,2, \ldots, n\}$.

Proof. Assume that $M$ is $N-\mathcal{I}_{F}-H$-supplemented. By Theorem 3.3, $M_{i}$ is $N$ - $\mathcal{I}_{F \cap M_{i}}-H$-supplemented for all $i \in\{1,2, \ldots, n\}$. Conversely, let $\phi$ be a homomorphism from $M$ to $N$. Consider $\phi=\left(\phi_{i}\right)_{i=1}^{n}$ where $\phi_{i} \in$ $\operatorname{Hom}_{R}\left(M_{i}, N\right)$ and $i \in\{1,2, \ldots, n\}$. Since $M_{i}$ is $N-\mathcal{I}_{F \cap M_{i}}-H$-supplemented, there exists a direct summand $D_{i}$ of $N$ such that $\phi_{i}\left(F \cap M_{i}\right) \beta^{*} D_{i}$ for all $i \in\{1,2, \ldots, n\}$. Using [3, Proposition 2.11] and this fact that $\phi(F)=$ $\sum_{i=1}^{n} \phi_{i}\left(F \cap M_{i}\right)$, we conclude that $\phi(F) \beta^{*} \sum_{i=1}^{n} D_{i}$. As $N$ has the SSP, $\sum_{i=1}^{n} D_{i}$ is a direct summand of $N$. Hence $M$ is $N-\mathcal{I}_{F^{-}} H$-supplemented. 
Proposition 3.7. Let $M=M_{1} \oplus M_{2}$ be a module, where $M_{1}$ and $M_{2}$ are fully invariant submodules of $M$. If $M$ is $\mathcal{I}_{M_{i}}-H$-supplemented for $i=1,2$,

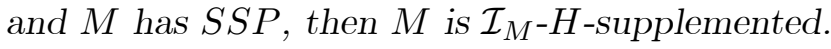

Proof. Let $\phi \in \operatorname{End}_{R}(M)$.Then, by assumption, there are direct summands $D_{1}$ and $D_{2}$ of $M$ such that $\phi\left(M_{1}\right) \beta^{*} D_{1}$ and $\phi\left(M_{2}\right) \beta^{*} D_{2}$. Note that, since $M$ has SSP, then $D_{1}+D_{2}$ is a direct summand of $M$. By [3, Proposition 2.11], $\phi(M) \beta^{*} D_{1}+D_{2}$. Therefore $M$ is $\mathcal{I}_{M}$ - $H$-supplemented.

Theorem 3.8. Let $M=M_{1} \oplus M_{2}$ be a duo module and let $F$ be a submodule of $M$. Then $M$ is $\mathcal{I}_{F-H}-H$-supplemented if and only if $M_{i}$ is $\mathcal{I}_{F \cap M_{i}}{ }^{-}$ $H$-supplemented for $i=1,2$.

Proof. $(\Rightarrow)$ By Corollary 3.5. $(\Leftarrow)$ Assume $M_{1}$ is $\mathcal{I}_{F \cap M_{1}}-H$-supplemented and $M_{2}$ is $\mathcal{I}_{F \cap M_{2}}-H$-supplemented. Let $\pi_{i}$ be the projection of $M$ on $M_{i}$ and let $j_{i}$ be the inclusion map from $M_{i}$ to $M$ for $i=1,2$. Assume that $f$ is an endomorphism of $M$. Since $M_{1}$ is $\mathcal{I}_{F \cap M_{1}}-H$-supplemented, there exists a direct summand $N_{1}$ of $M_{1}$ such that $M_{1}=N_{1}+X$ if and only if $M_{1}=\pi_{1} f j_{1}\left(F \cap M_{1}\right)+X$ for any submodule $X$ of $M_{1}$ and since $M_{2}$ is $\mathcal{I}_{F \cap M_{2}}-H$-supplemented, there exists a direct summand $N_{2}$ of $M_{2}$ such that $M_{2}=N_{2}+Y$ if and only if $M_{2}=\pi_{2} f j_{2}\left(F \cap M_{2}\right)+Y$ for any submodule $Y$ of $M_{2}$. We claim that $M=N_{1} \oplus N_{2}+Z$ if and only if $M=f(F)+Z$ for any submodule $Z$ of $M$. Note that $f(F)=\pi_{1} f\left(F \cap M_{1}\right)+\pi_{2} f\left(F \cap M_{2}\right)$, because, $F=\left(F \cap M_{1}\right) \oplus\left(F \cap M_{2}\right)$ implies $f(F)=f\left(F \cap M_{1}\right) \oplus f\left(F \cap M_{2}\right)=$ $\pi_{1} f\left(F \cap M_{1}\right)+\pi_{2} f\left(F \cap M_{2}\right)$. Let $M=N_{1} \oplus N_{2}+Z$. Then

$$
\begin{aligned}
& M_{1}=N_{1}+\left(M_{1} \cap\left(N_{2}+Z\right)\right)=\pi_{1} f j_{1}\left(F \cap M_{1}\right)+\left(M_{1} \cap\left(N_{2}+Z\right)\right) \\
& =M_{1} \cap\left[\pi_{1} f j_{1}\left(F \cap M_{1}\right)+\left(N_{2}+Z\right)\right] .
\end{aligned}
$$

Thus $M_{1} \leq \pi_{1} f j_{1}\left(F \cap M_{1}\right)+\left(N_{2}+Z\right)$. Then $M_{1} \leq \pi_{1} f\left(F \cap M_{1}\right)+Z$, because, let $m_{1}=\pi_{1} f(y)+n_{2}+z_{0}$, where $m_{1} \in M_{1}, y \in F \cap M_{1}, n_{2} \in N_{2}$ and $z_{0} \in Z$. Since $Z=\left(Z \cap M_{1}\right) \oplus\left(Z \cap M_{2}\right), z_{0}=z_{1}+z_{2}$ where $z_{1} \in Z \cap M_{1}$ and $z_{2} \in Z \cap M_{2}$. Then $m_{1}=\pi_{1} f(y)+z_{1}$ and so $M_{1} \leq \pi_{1} f\left(F \cap M_{1}\right)+Z$. Similarly, $M_{2} \leq \pi_{2} f\left(F \cap M_{2}\right)+Z$. Thus $M=\pi_{1} f\left(F \cap M_{1}\right)+\pi_{2} f\left(F \cap M_{2}\right)+Z$. Therefore $M=f(F)+Z$.

Conversely, assume that $M=f(F)+Z$. Then $M=\pi_{1} f\left(F \cap M_{1}\right)+$ $\pi_{2} f\left(F \cap M_{2}\right)+Z$. By modularity,

$$
M_{1}=\pi_{1} f\left(F \cap M_{1}\right)+\left[M_{1} \cap\left(\pi_{2} f\left(F \cap M_{2}\right)+Z\right)\right]
$$


and so

$$
M_{1}=N_{1}+\left[M_{1} \cap\left(\pi_{2} f\left(F \cap M_{2}\right)+Z\right)\right]=M_{1} \cap\left(N_{1}+\pi_{2} f\left(F \cap M_{2}\right)+Z\right) .
$$

Thus $M_{1} \leq N_{1}+\pi_{2} f\left(F \cap M_{2}\right)+Z$. Therefore $M_{1} \leq N_{1}+Z$. Similarly, $M_{2} \leq N_{2}+Z$. Hence $M=\left(N_{1} \oplus N_{2}\right)+Z$.

Proposition 3.9. Let $F$ be a fully invariant submodule of a module $M$. Assume $\phi(F)$ has a supplement that is a direct summand of $M$ for every $\phi \in \operatorname{End}_{R}(M)$ such that whenever $M=M_{1} \oplus M_{2}$ then $M_{1}$ and $M_{2}$ are relatively projective. Then $M$ is an $\mathcal{I}_{F}-H$-supplemented module.

Proof. Let $\phi \in \operatorname{End}_{R}(M)$. By hypothesis, there exists a decomposition $M=M_{1} \oplus M_{2}$ such that $M=\phi(F)+M_{2}$ and $\phi(F) \cap M_{2} \ll M_{2}$ for some submodules $M_{1}$ and $M_{2}$ of $M$. Since $M_{1}$ is $M_{2}$-projective, by [9, Lemma 4.47], we get $M=N \oplus M_{2}$ for some submodule $N$ of $M$ such that $N \leq \phi(F)$. Then $\phi(F)=N \oplus\left(M_{2} \cap \phi(F)\right)$. Let $X \leq M$ with $M=\phi(F)+X$. Then $M=N+\left(M_{2} \cap \phi(F)\right)+X$. As $M_{2} \cap \phi(F) \ll M_{2}, M=N+X$. Therefore $M=N+X$ if and only if $M=\phi(F)+X$. Hence $M$ is $\mathcal{I}_{F}-H$-supplemented.

\section{References}

[1] T. Amouzegar, "A generalization of lifting modules", Ukrainian mathematical journal, vol. 66, no. 11, pp. 1654-1664, Apr. 2015, doi: $10.1007 / \mathrm{s} 11253-015-1042-\mathrm{z}$

[2] T. Amouzegar and A. R. Moniri Hamzekolaee, "Lifting modules with respect to images of a fully invariant submodule", Novi Sad journal mathematical, vol. 50, no. 2, pp. 41-50, 2020, doi: 10.30755/ NSJOM.09413

[3] G. F. Birkenmeier, F.T. Mutlu, C. Nebiyev, N. Sokmez and A. Tercan, "Goldie*-supplemented modules", Glasgow mathematics journal, vol. 52, no. A, pp. 41-52, Jun. 2010, doi: 10.1017/ S0017089510000212

[4] J. Cark, C. Lomp, N. Vanaja, and R. Wisbauer, Lifting modules supplements and projectivy in module theory. Basel: Birkhäuser, 2006, doi: $10.1007 / 3-7643-7573-6$ 
[5] A. Ghorbani and M. R. Vedadi, "Epi-retractable modules and some applications", Bulletin Iranian Mathematics Society, vol. 35, no. 1, pp. 155-166, 2009. [On line]. Available: https:/ / bit.ly/3hiLmRO

[6] D. Keskin, "Finite direct sums of D1-modules", Turkish journal mathematical, vol. 22, pp. 85-91, 1998. [On line]. Available: https:// bit.ly/2KxT5zA

[7] D. Keskin, M. J. Nematollahi, and Y. Talebi, "On H-supplemented modules", Algebra colloquium, vol. 18, no. s01, pp. 915-924, 2011, doi: $10.1142 /$ S1005386711000794

[8] G. Lee, S. T. Rizvi and C. S. Roman, "Dual Rickart modules", Communications in algebra, vol. 39, no. 11, pp. 4036-4058, Nov. 2011, doi: $10.1080 / 00927872.2010 .515639$

[9] S. H. Mohamed and B. J. Müller, Continuous and discrete modules, Cambridge: Cambridge University Press, 1990, doi: $10.1017 /$ CBO9780511600692

[10] A. R. Moniri Hamzekolaee, Y. Talebi, A. Harmanci and B. Ungor, "A new approach to $H$-supplemented modules via homomorphisms", Turkish journal mathematical, vol. 43, pp. 1941-1955, Jul. 2018, doi: 10.3906/ mat-1709-74

[11] A. C. Özcan, A. Harmanci, and P. F. Smith, "Duo modules", Glasgow mathematics journal, vol. 48, no. 3, pp. 533-545, Sep. 2006, doi: $10.1017 /$ S0017089506003260

[12] Y. Talebi and N. Vanaja, "The torsion theory cogenerated by M-small modules", Communications in algebra, vol. 30, no. 3, pp. 1449-1460, 2002, doi: 10.1080/00927870209342390

[13] Y. Talebi, R. Tribak, and A. R. Moniri Hamzekolaee, "On H-cofinitely supplemented modules", Bulletin Iranian Mathematical Society, vol. 39, no. 2, pp. 325-346, 2013. [On line]. Available: https:// bit.ly/2KIiCG5

[14] R. Wisbauer, Foundations of module and ring theory, Reading: Gordon and Breach, 1991. [On line]. Available: https:// bit.ly/37OJY6d 